\title{
Physical Rehabilitation of 5-10-Year-Old Children With Bronchial Asthma at the Stationary Stage
}

\author{
Bakay I.N. ${ }^{1,{ }^{*}}$ Konstantinova N.P. ${ }^{2}$ Shimanovskaya Y.V. ${ }^{2}$ Sarychev A.S. ${ }^{3}$ \\ ${ }^{1}$ Faculty of Physical Culture, Department of Adaptive Physical Culture and Recreation, Russian State Social University, \\ Moscow, Russia \\ ${ }^{2}$ Faculty of Social Work, Department of Social Work, Russian State Social University, Moscow, Russia \\ ${ }^{3}$ Department of Hygiene and Medical Ecology, Northern State Medical University, Arkhangelsk, Russia \\ *Corresponding author. Email: irinasyazina@mail.ru
}

\begin{abstract}
This article discusses the peculiarities of the method of physical rehabilitation of (5-10)-year-old children with bronchial asthma at the stationary stage of recovery. The social significance of the problem of rehabilitation of children with chronic bronchopulmonary diseases, including bronchial asthma, is due to the high prevalence, severe, progressive course of the process, adverse impact on the growth and development of the child, the possibility of early disability and deterioration in the quality of life of the child. Despite the significant success achieved in the drug therapy of bronchial asthma in children, many aspects of the treatment of this disease remain unresolved. In this regard, a method of physical rehabilitation for children with bronchial asthma at the stationary stage of rehabilitation has been developed and presented for publication.
\end{abstract}

Keywords: bronchial asthma, physical rehabilitation, exercise therapy, children, bronchopulmonary diseases

\section{INTRODUCTION}

Bronchial asthma is not only a medical problem, but also an urgent socially significant problem, since the disease leads to early disability and a decrease in the child's "quality of life" indicators. The main reason that leads to disability and mortality in children with bronchial asthma is respiratory failure, which is mainly due to the development of the airflow restriction syndrome and the syndrome of early expiratory airway closure [4, 7].

A number of non-drug methods have been developed, among which physical rehabilitation methods play a leading role: a set of measures aimed at preventing disability, improving the quality of life of a sick child, restoring health as a result of an existing disease.

In connection with the above, the development of a method of physical rehabilitation for bronchial asthma in children 5-10 years old at the stationary stage is an urgent task of restorative medicine.

In the complex of the treatment of children with bronchial asthma, means of physical rehabilitation are a powerful factor in the healing effect on the child's body. In the process of physical rehabilitation, the onset of remission occurs due to the mobilization of the child's own compensatory capabilities. Based on this, these means are the most physiologically correct, and their correct use does not cause complications, which is very important for children $[2,3,5,6,9]$.

Regular, therapeutic, special exercises for the respiratory system are the core of asthma therapy. Therapeutic physical exercises help to resist the development of emphysema, strengthens the respiratory muscles, increases the elasticity of lung tissue, promotes the discharge of bronchial secretions. The ultimate goal of physical rehabilitation is the adaptation of the child's body to the general level of physical activity, increasing efficiency, improving physical development, expanding the functional capabilities of the body as a whole $[1,8,10]$.

\section{METHODS AND MATERIALS}

The study was conducted from September to December 2019 at the Moscow Regional Research Clinical Institute named after M.F. Vladimirsky.

The study involved 20 children aged 5-10 years with a diagnosis of bronchial asthma with a predominance of an allergic component. The control group consisted of 10 children who underwent physical rehabilitation according to the hospital method, including 6 boys and 4 girls, with an average age of $7.4 \pm 0.84$ years. The experimental group consisted of 10 children, with whom the developed method of physical rehabilitation was carried out, including 6 boys and 4 girls with an average age of $7.5 \pm 0.85$ years.

To solve the set tasks, the following research methods were used:

1. Analysis of scientific and methodological literature (national and international sources);

2. Assessment of the functional state of the respiratory system: respiratory rate (RR), chest excursion (CE), lung capacity (LC), timed inspiratory capacity (TIC), timed expiratory capacity (TEC), the complex indicator of the 
endurance of the respiratory system (CIERS), pneumotachometry (PTM);

3. Pedagogical experiment;

4. Methods of mathematical statistics.

The developed method of physical rehabilitation of 5-10-year-old children with bronchial asthma at the stationary stage consisted of three periods: preparatory (6 days), main (10 days) and final (5 days). The technique was designed for 21 days (3 weeks).

The goal of the proposed method of physical rehabilitation of 5-10-year-old children with bronchial asthma at the stationary stage was to normalize the function of the respiratory system, reduce spasms of bronchioles, bronchi, increase chest mobility, and increase resistance to external factors.

Tasks of the rehabilitation method:

- improving the functions of the respiratory and cardiovascular systems;

- $\quad$ increasing the adaptation of the body to stress;

- improving the function of external respiration, drainage function and ventilation of the bronchopulmonary system;

- $\quad$ strengthening of the main and auxiliary respiratory muscles;

- $\quad$ prevention of exacerbation of the disease;

- improvement of the psycho-emotional state;

- normalization of immunity.

The following means and forms were included in the methodology of physical rehabilitation of 5-10-year-old children with bronchial asthma at the stationary stage: therapeutic exercises, dosed walking, terrenkur (dosed ascent), hydrokinesic therapy, therapeutic massage, physiotherapeutic procedures, speleotherapy.

Therapeutic gymnastics for 5-10-year-old children with bronchial asthma was carried out on the basis of complexes of general developmental exercises, special exercises for training the respiratory system.

In the preparatory part of the lesson, relaxation exercises were performed, then self-massage techniques, exercises for the tongue, pharyngeal exercises were delivered.

In the main part of the lesson, they switched to breathing exercises (static and dynamic, sound involving and those with an extended exhalation) in the initial lying and sitting positions. In this position, the greatest relaxation of the muscles was achieved, and the abdominal and lower-rib muscles were included in the training. The exercises were structured in such a way that at first the skills of correct breathing were involuntarily fixed as a result of training, and then they were automated and became habitual. The exercises took into account the anatomy and physiology of the speech apparatus, the process of breathing regulation, with an emphasis on exhalation.
In the final part of the lesson, relaxation exercises were performed in which muscle relaxation occurred.

In the exercises, the following principle was used: a natural, reflex short breath, and then a long exhalation with a sound, a phrase, and a small poetic text.

The expiratory duration increased gradually and was not forced.

In the preparatory period, the exercise complexes were presented in groups. When performing exercises in the supine position, the greatest number of muscles relaxed, cramps in the neck, shoulders, and back were removed. These exercises contributed to the inclusion of the lower abdominal and lumbar muscles in the breathing process and the formation of the diaphragmatic type of breathing.

Exercises in a standing and sitting position on a chair (leaning, half- leaning, upward arm movement) relieve tension from the muscles of the neck, shoulders, upper chest and, indirectly, from the intraopharyngeal muscles. In addition, half-leaning and leaning relieve the upper clavicular breathing. When squatting, the breath is reflexively produced in the lower parts of the lungs.

In all exercises, the movement of the body during inhalation was energetic, the inhalation was made reflexively, attention was not fixed on the inhalation process, the degree of inhalation was regulated by the movement itself. Attention was fixed only on exhalation and speech, in which the movements of the arms, body and legs were slow, smooth, without jerks and were coupled with a fixed exhalation and exhalation with sounds and speech.

In the main period, exercises with a ball were added. In the exercises, when swinging the hand with the ball, a quick and at the same time deep breath, imperceptible for the child, occurred, the tension of the neck and shoulders was relieved. Ball exercises created a positive emotional attitude, which contributed to faster assimilation of exercises and the formation of necessary skills. The exercises were performed with a tennis ball as well as a large ball. Classes were held not only in the physical therapy hall, but also in the fresh air.

In the final period of rehabilitation, a set of special exercises and active games was additionally carried out to enhance inhalation.

Game breathing exercises were also conducted.

1. "Crow". Sitting comfortably on a chair with arms outstretched at the sides, the child pulls out the straightened arms through the sides upwards on inhalation, and on exhalation lowers them down, pronouncing a drawn-out "carrr".

2. "Beetle". In a similar position, the child spreads the arms to the sides, holds them overhang, and also spreads their legs as wide as possible. During inhalation, the body turns to the side, while exhaling, it turns straight again, at this time the child imitates the buzzing of a flying beetle: "zzhzh".

3. "Geese". While still sitting, the child places the fingers on the shoulder girdle. Inhaling, tilts the body parallel to the floor exhaling, straightens, pronouncing "gaa" or "guu". 
4. "Crane". Standing up straight, the child lowers the arms along the body, then, inhaling, pulls them up through the sides exhaling, lowers them again, reproducing the sound "urrr".

5. "Stork". The child puts his feet shoulder-width apart, pushes the hands to the sides. During inhalation, they alternately pull the bent legs upwards, while exhaling they put them on the floor while pronouncing "shshsh".

6. Exercise "Good morning". This exercise is performed while lying down. Bring your knees close to your chest while breathing out slowly through your mouth. Straighten your legs, relax.

7. Exercise "Balloon". Stand up straight, inhale with your nose, sticking out your stomach strongly. Exhale sharply drawing in your stomach.

8. Exercise "Hugs". Stand on your toes, stretch your arms to the sides, parallel to the floor, bend forward. Exhale deeply, at the same time rapidly cross your arms on your chest, as if hugging yourself, hitting your shoulder blades with your palms, spread your arms again and repeat the exercise, continuing to exhale. Inhale and return to starting position.

9. Exercise with a straw. Put a jar of water in front of the child, give a straw, for example, from baby juice. Take a very deep breath through the tube, and then gradually exhale into the water.

When prescribing active games of a special orientation for the rehabilitation of bronchial asthma, the age, individual characteristics of the child's personality, mental development, the degree of physical fitness and, necessarily, the specificity of the disease (the degree of severity, the nature of the reaction to the load, the phase of the patient's condition - stable, unstable remission, etc.) were taken into account.

One lesson lasted 25-30 minutes.

The massage was carried out in the first half of the day, with a break of 1.5-2 hours after eating. The session lasted from 12 to 15 minutes. The procedure was as follows:

1. The child is sitting, while the masseur stands behind them. The child should relax, breathe evenly, calmly. For the first 2-3 minutes the back, front and side of the chest, back of the neck is massaged by gently stroking and rubbing the skin.

2. The child lies on their stomach. The muscles of the back, intercostal spaces, the area above the shoulder blades are massaged. Such techniques as kneading, rubbing, stroking are used. Rubbing (rectilinear, spiral) is performed in the thoracic spine towards the neck, then circular from the spine to the shoulder blade. After that, stroking and rubbing the entire surface of the back are performed again, and the muscles are kneaded.

3. The child rolls over onto their back. The massage combines stroking and kneading, after which the area around the collarbone is rubbed, the pectoralis major muscle is kneaded and stroked. The therapist places their palms on the ribs below the diaphragm and asks the child to breathe out each time they press on them. Three or four areas are distinguished, the pressure is applied 2-4 times in turn.
The session ends with chest massage with an emphasis on rubbing the intercostal spaces.

The course of treatment is 15 procedures daily.

In the rehabilitation of 5-10-year-old children with bronchial asthma, aeroionotherapy was additionally used at the stationary stage.

An Elion-131 M device was used for aeroionotherapy. Recommended dose: 50 billion per 1 procedure, "intensive" mode on; distance from the apparatus was $0.5 \mathrm{~m}$, procedure duration was 16 minutes. The course included 15 daily procedures or 2 procedures a day with an interval of 1.5-2 hours. The course consisted of 10 daily procedures with a duration of 30 minutes.

Also, the complex methodology included hydrokinesis therapy classes, which were carried out in a children's shallow pool.

The course of hydrokinesis therapy consisted of 2 periods: preparatory and main.

In the preparatory period, general strengthening and tempering activities were carried out, and in the main period, general strengthening, tempering and therapeutic activities were carried out, namely, breathing exercises, water tempering procedures, physical exercises in water.

At the first lessons in the pool, children were taught diaphragmatic breathing and the stereotype of correct breathing. Therapeutic exercises in the pool were carried out daily by a group method.

The average duration of the therapeutic gymnastics procedure in the pool was 30-35 minutes; during the stay in the hospital, a child attended 15 classes.

In the final period of rehabilitation, a terrenkur was carried out, i.e. walking along the zones that is dosed in terms of distance, pace and angle of inclination, which has a training effect on the muscular, cardiovascular and respiratory systems.

The terrenkur was starting with walking horizontally or with ascents at a slight angle, at a calm pace, with a rhythmical step.

While walking, it was important to monitor the posture and follow the breathing guidelines. On a flat road, the inhale should correspond to 2-4 steps, and exhale should correspond to 3-5 steps. When ascending, the breathing should be more frequent 2-3 steps for inhale and 3-4 for exhale, the steps should be shorter. The breathing should be nasal and mixed (with expansion of the chest and a slight protrusion of the abdomen on inhalation).

Terrenkur walks were carried out regularly every day in the morning. Taking into account the age of the children, the routes including stops with game tasks were developed. The terrenkur route included not only specially created centers, but also a sports ground, a play area on asphalt, a health path, an ecological path, flower beds, and other possible objects on the territory of the hospital.

Speleotherapy was carried out in a speleochamber. The basis of the therapeutic artificial microclimate was a highly dispersed sodium chloride aerosol sprayed with a special apparatus in a room with a constant temperature $\left(20-23{ }^{\circ} \mathrm{C}\right)$ and humidity $(40-60 \%)$ of air. $\mathrm{NaCl}$ aerosol has pronounced hypoallergenic and hypobacterial properties; penetrating into the lungs, it prevents the 
development of infectious pathogens, promotes extinction of inflammatory processes, dilutes the mucous secretion of the respiratory tract, thereby restoring the physiological properties of epithelial tissue. Recovery in a speleo chamber has a mucolytic, sanogenic, anti-inflammatory, bronchodrainage, immunomodulatory effect on the tissues of the respiratory system.

On the first day, the children spent 5 minutes in the artificial speleotherapy chamber, 10 minutes on the second day, 15 minutes on the third day and 20 minutes on the fourth day. Later, if there were no adverse reactions, the children stayed in the chamber for 25-30 minutes every day. During speleotherapy, no special breathing exercises were used, children were asked to sit, play and breathe freely so that the procedures in the speleo chamber were interesting. The course of treatment consisted of 15 procedures (10 procedures in the main period and 5 in the final period).

Table 1 Comparative analysis of the average indicators of the functional state of the respiratory system in the control and experimental groups before the experiment
The use of the developed methodology for physical rehabilitation of 5-10-year-old children with a diagnosis of bronchial asthma at the stationary stage contributed to the improvement of the functional state of the respiratory system of young patients. At the beginning of the experiment, testing was carried out for all the studied parameters. The test results are presented in Table 1.

Intergroup comparison of indicators revealed that before the experiment, the control and experimental groups did not have significant differences, which proves the homogeneity of the groups.

To assess the effectiveness of physical rehabilitation programs after the experiment, we carried out a comparative analysis of the average indicators of the functional state of the respiratory system in the studied groups, which is presented in Table 2 .

\begin{tabular}{|l|c|c|c|}
\hline \multirow{2}{*}{ Tests } & \multicolumn{2}{|c|}{ Study group } & \multirow{2}{*}{ p } \\
\cline { 2 - 4 } & CG $(\mathbf{n}=10) \mathbf{M} \pm \boldsymbol{\sigma}$ & $22.40 \pm 0.69$ & $\geq 0.05$ \\
\hline RR, min $^{-1}$ & $22.90 \pm 0.73$ & $3.69 \pm 0.23$ & $\geq 0.05$ \\
\hline CE, cm & $3.72 \pm 0.23$ & $1259.50 \pm 33.5$ & $\geq 0.05$ \\
\hline LC, ml & $1274.20 \pm 47.3$ & $13.20 \pm 0.54$ & $\geq 0.05$ \\
\hline TIC, s & $13.00 \pm 0.50$ & $12.20 \pm 0.56$ & $\geq 0.05$ \\
\hline TEC, s & $12.30 \pm 0.55$ & $12.70 \pm 0.33$ & $\geq 0.05$ \\
\hline CIERS, s & $12.65 \pm 0.29$ & $1.33 \pm 0.05$ & $\geq 0.05$ \\
\hline PTM, 1/s & $1.38 \pm 0.04$ & & \\
\hline
\end{tabular}

Table 2 Comparative analysis of the average indicators of the functional state of the respiratory system in the control and experimental groups after the experiment

\begin{tabular}{|l|c|c|c|}
\hline \multirow{2}{*}{ Tests } & \multicolumn{2}{|c|}{ Study group } & \multirow{2}{*}{ p } \\
\cline { 2 - 4 } & CG $(\mathbf{n}=10) \mathbf{M} \pm \sigma$ & $17.10 \pm 0.37$ & $<0.05$ \\
\hline RR, min $^{-1}$ & $20.40 \pm 0.61$ & $4.96 \pm 0.13$ & $<0.05$ \\
\hline CE, cm & $3.92 \pm 0.20$ & $1882.30 \pm 62.7$ & $<0.05$ \\
\hline LC, $\mathrm{ml}$ & $1430.10 \pm 70.3$ & $23.10 \pm 1.01$ & $<0.05$ \\
\hline TIC, s & $16.60 \pm 0.80$ & $19.10 \pm 0.79$ & $<0.05$ \\
\hline TEC, s & $14.90 \pm 0.46$ & $21.10 \pm 0.67$ & $<0.05$ \\
\hline PTERS, s & $15.75 \pm 0.52$ & $1.91 \pm 0.07$ & $<0.05$ \\
\hline
\end{tabular}


The intergroup comparison of indicators revealed that after the experiment, the control and experimental groups had a significant difference in all the studied indicators. Let us consider the change in indicators in more detail.

Respiratory rate indices in the experimental group decreased by $5.3 \mathrm{~min}^{-1}(23.66 \%)$; in the control group the respiratory rate decreased by $2.5 \mathrm{~min}^{-1}(10.92 \%)$

The change in the respiration rate (RR) revealed during the experiment indicates the effectiveness of the breathing exercises in the course of therapeutic gymnastics in the classroom with children of the experimental group.

After rehabilitation measures, the indicators of chest excursion (CE) in the experimental group increased by $1.27 \mathrm{~cm}(34.42 \%)$; in the control group it increased by $0.2 \mathrm{~cm}(5.38 \%)$.

The resulting change indicates the effectiveness of the therapeutic exercises with breathing exercises and speleotherapy.

An increase in the indicators of lung capacity (LC) occurred in the control group by $155.9 \mathrm{ml}(12.24 \%)$, and in the experimental group the increase was $622.8 \mathrm{ml}$ $(49.45 \%)$.

The increase in LC of the children of the experimental group was more pronounced due to the use of aeroionotherapy and breathing exercises.

The analysis of the test results showed that TIC in the experimental group improved by 9.9 seconds, which is $75 \%$, and in the control group it improved by 3.6 seconds, which is $27.69 \%$.

It was revealed that TEC of the children of the experimental group improved by 6.9 seconds, which is $56.56 \%$, the indicators of the control group improved by 2.6 seconds, which is $21.14 \%$.

The change in the TEC is clear.

A positive change in the indicators of breath holding tests in children of the experimental group indicates the effectiveness of the use of physical exercises in water.

The increase in indicators of endurance of the respiratory system in the experimental group was 8.4 seconds $(66.14 \%)$, in the control group the indicators increased by 3.1 seconds $(24.51 \%)$.

A more pronounced change in the complex indicator of the endurance of the respiratory system in children of the experimental group occurred due to the use of dosed walking and terrenkur.

The increase in the parameters of the peak forced expiratory flow rate occurred both in the control and in the experimental group. A more pronounced change is observed in the experimental group of $0.58 \mathrm{l} / \mathrm{s}$ (43.61\%), while in the control group the increase was $0.13 \mathrm{l} / \mathrm{s}$ $(9.42 \%)$.

The increase in pneumotachometry indicators was due to special breathing exercises to enhance the expiratory force.

In connection with the above, it can be argued that the developed method of physical rehabilitation of 5-10-year children with a diagnosis of bronchial asthma at the stationary stage is quite effective.

\section{CONCLUSION}

In the process of research work on the use of means and methods of physical rehabilitation for 5-10-year-old children with bronchial asthma, a method of physical rehabilitation at a stationary stage, lasting 21 days, was developed. The technique was divided into three periods: preparatory (6 days), main (10 days), and final (5 days). The physical rehabilitation methodology included morning hygienic exercises, therapeutic exercises with breathing exercises, therapeutic massage, hydrokinesis therapy, dosed walking, health path, physiotherapy, speleotherapy.

The use of the developed methodology for physical rehabilitation of 5-10-year-old children diagnosed with bronchial asthma at the stationary stage contributed to the improvement of the functional state of the respiratory system of patients:

- RR indicator in the experimental group improved by $23.66 \%$, and by $10.92 \%$ in the control group $(\mathrm{p}<0.05)$;

- $\quad$ E indices improved in the experimental group by $34.42 \%$, and by $5.38 \%$ in the control group $(\mathrm{p}<0.05)$;

- LC indicators changed in the experimental group by $49.45 \%$ and by $12.24 \%$ in the control group $(\mathrm{p}<0.05)$;

- TEC indicators improved in the control group by $75 \%$ and by $27.69 \%$ in the experimental group $(\mathrm{p}<0.05)$;

- $\quad$ TIC indicators in the experimental group improved by $56.56 \%$ and $21.14 \%$ in the control group by $(\mathrm{p}<0.05)$;

- CIERS complex indicator in the experimental group improved by $66.14 \%$ and by $24.51 \%$ in the control group $(\mathrm{p}<0.05)$;

- PTM indicator in the experimental group improved by $43.61 \%$ and by $9.42 \%$ in the control $(\mathrm{p}<0.05)$.

According to the results of the study, the comparison of the indicators of the respiratory system functional state showed the presence of significant differences $(p<0.05)$ in all indicators between the experimental and control groups, which indicates the effectiveness of the developed method of physical rehabilitation for this category of children.

Based on the results of the work, the following practical recommendations can be made:

1. The method of physical rehabilitation of 5-10-yearold children with bronchial asthma in the stationary stage should consist of three periods: preparatory, main and final. In the preparatory period, the main tasks are to improve the functions of the respiratory system and 
increase adaptation. The program is recommended to include morning hygienic exercises, therapeutic exercises, dosed walking and hydrokinesis therapy.

In the main period, to improve the drainage function and ventilation of the bronchopulmonary system, therapeutic massage and physiotherapy procedures are added.

In the final period, when carrying out rehabilitation measures, an emphasis is placed on strengthening the main and auxiliary respiratory muscles and preventing an exacerbation of the disease, and in addition to the existing activities, active games are involved, dosed walking is replaced by terrenkur.

2. When compiling a set of exercises for therapeutic gymnastics, it is necessary to take into account the age characteristics of children. Therapeutic exercises for 5-10year-old children with bronchial asthma should be carried out on the basis of complexes of general developmental exercises, breathing exercises, game exercises for breathing training and special exercises. The simplest exercises should be included in therapeutic exercises. The basic principle in the implementation of the exercises is the implementation of a natural reflex short inhalation, and then a long exhalation with sound, phrase, or poetic text. The expiration time should be increased gradually. Between breathing exercises with the pronunciation of sounds, it is imperative to include a pause for rest to relax the muscles. The dosage of exercise is $4-12$ times, the pace is slow and medium.

3. During the massage, it is recommended to carry out the exercises daily in the position in which the child can breathe comfortably.

4. When conducting a terrenkur, it is necessary to perform breathing exercises at the beginning and at the end of the lesson, as well as at the stops of the route. Tasks at stops should be ceased if the first signs of an attack of suffocation appear: uneven breathing, spasm, cough. Intensive running, exercise without interruption to establish calm breathing are not allowed. It is contraindicated to practice outdoors in adverse weather conditions (too cold weather, rain, wind), since any hypothermia can cause an attack of suffocation.

\section{REFERENCES}

[1] I.N. Bakay, V.P. Kartashev, N.V. Karpova, A.V. Kornev, A.N. Studneva, Basics of sanatoriumresort rehabilitation for persons with asthma, Res. J. of pharmac., biology. and chem. Sci. 6(9) (2018) 1009-1015.

[2] D.G. Kazanbekov, K.A. Masuev, K.M. Alieva, The effectiveness of methods of rehabilitation of patients with bronchial asthma, Bull. of new med. technolog. 3 (2014) 120-121.

[3] D.M. Obukhova, Yu.O. Ivanova, Physical rehabilitation of children with bronchial asthma, Actual sci. res. in the modern world 4-3 (2017) 115-116.

[4] E.A. Vishneva, Actual tactics of management of children with bronchial asthma, Pediatric Pharmacol. 14(6) (2017) 119.

[5] E.S. Krasavina, S.I. Alekseeva, Physical rehabilitation of 6-9-year-old children with bronchial asthma by adaptive physical exercises, Phys. culture. Sport. Tourism. Motion recreat. 3(1) (2018) 58-62.

[6] I.N. Bakai, N.V. Karpov, V.P. Kartashev, A.N. Studneva, Health-developing technologies in physical rehabilitation of primary school children with autonomic disorders, Theory and pract. of phys. culture 5 (2019) 56-59.

[7] N.A. Geppe, N.G. Kolosov, The value of national guidelines in the choice of methods for treating bronchial asthma in children, Attending Physician 2-2013 (2018) 69.

[8] N.M. Valeev, N.L. Ivanova, Physical rehabilitation of patients with respiratory diseases: training manual, Physical culture, Moscow, 2009, 105 p.

[9] N.P. Tsygulya, I.N. Syazin, B.A. Marshinin Occupational therapy in social work, Teaching guide, APK and PPRO, Moscow, 2012, 56 p.

[10] I.K. Volkov et al. (sci. eds), Bronchial asthma in children. Treatment strategy and prevention, 5rd ed., revised and supplemented, Original-maket, Moscow, 2016, 182 p. 